\title{
Speckle Reduction in Optical Coherence Tomography by Image Registration and Matrix Completion
}

\author{
Jun Cheng ${ }^{1}$, Lixin Duan ${ }^{1}$, Damon Wing Kee Wong ${ }^{1}$, Dacheng Tao ${ }^{2}$, \\ Masahiro Akiba ${ }^{3}$, and Jiang $\mathrm{Liu}^{1}$ \\ 1 Institute for Infocomm Research, Agency for Science, \\ Technology and Research, Singapore \\ \{jcheng, duanlx, wkwong, jliu\}@i2r.a-star.edu.sg \\ 2 University of Technology, Sydney, Australia \\ 3 Topcon Corporation, Tokyo, Japan
}

\begin{abstract}
Speckle noise is problematic in optical coherence tomography (OCT). With the fast scan rate, swept source OCT scans the same position in the retina for multiple times rapidly and computes an average image from the multiple scans for speckle reduction. However, the eye movement poses some challenges. In this paper, we propose a new method for speckle reduction from multiply-scanned OCT slices. The proposed method applies a preliminary speckle reduction on the OCT slices and then registers them using a global alignment followed by a local alignment based on fast iterative diamond search. After that, low rank matrix completion using bilateral random projection is utilized to iteratively estimate the noise and recover the underlying clean image. Experimental results show that the proposed method achieves average contrast to noise ratio 15.65 , better than 13.78 by the baseline method used currently in swept source OCT devices. The technology can be embedded into current OCT machines to enhance the image quality for subsequent analysis.
\end{abstract}

Keywords: Speckle, matrix completion, bilateral random projection.

\section{Introduction}

Optical coherence tomography (OCT) is a micrometer-scale, cross-sectional imaging modality for biological tissue. In OCT images, the structures are often obscured due to speckle noise. Recently, Topcon has developed DRI OCT-1, a swept source OCT for posterior imaging of the eye, utilizing a wavelength sweeping light source at $1,050 \mathrm{~nm}$ wavelength range. It has a fast scanning speed of 100,000 $A$-scans/second. In DRI OCT-1, the speckle noise in a single raw $B$-scan image often makes it challenging to examine the detailed structure. Fig. 1 shows an example of raw slice. Thus, speckle noise reduction is important to improve the image quality in the DRI OCT-1.

Besides OCT, speckle reduction has also been an important research topic in many other imaging modalities such as ultrasound, sonar, remote sensing, etc. In 


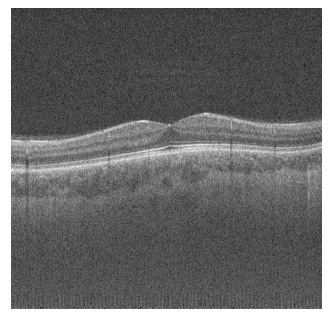

Fig. 1. A raw slice ( $B$-scan) of OCT image captured by DRI OCT-1

these fields, many speckle reduction algorithms have been proposed such as enhanced Lee filter [1, adaptive Wiener filter [2, anisotropic diffusion [3], speckle reduction anisotropic diffusion $(\mathrm{SRAD})$ [4, etc. Although these methods are efficient in removing speckle noise, they often remove some details simultaneously. Therefore, their practical usage is limited.

With the fast imaging speed, DRI OCT-1 utilizes a new strategy for speckle reduction by scanning the same or approximately the same position for multiple times. For example in a line scan mode in DRI OCT-1, a maximum of 96 scans can be obtained for the same line. Then an average image is computed from the 96 scans to generate a $B$-scan image. The averaging strategy expects the cancelling out of speckle noise from the different scans. It has been shown that averaging successfully reduces the noise [5. However, it has limitations. Firstly, the actual locations scanned are often shifted due to eye movement in the capture. This may lead to some changes in the underlying image. Although the changes are normally small, they hardly cancel out with each other. Secondly, the multiply-scanned slices are often not well aligned due to eye movement and image registration is required. However, the high amount of speckle may dominate the registration. In DRI OCT-1, a rigid sub-pixel registration algorithm [6] is used to register the slices. It minimizes the mean square intensity difference between one slice and its reference mainly through translation. However, it is not robust in presence of large speckle noise.

In this paper, we propose a new method for speckle reduction from multiple OCT slices. In this method, a preliminary speckle reduction is first applied on the raw unprocessed slices. Then, a non-rigid two-step registration is proposed to align the slices. After that, low rank matrix completion is proposed to compute the clean image. Because the multiple scans are usually obtained from very close but different locations, it is inevitable that the underlying images have some slight differences. The basic idea of low rank matrix completion is to formulate the $k^{t h}$ OCT slice $I_{k}$ as a sum of its underlying clean image part $l_{k}$, a sparse part $s_{k}$ due to eye movement and a noise part $n_{k}$ due to speckle noise:

$$
I_{k}=l_{k}+s_{k}+n_{k}
$$

Define $X=\left[\tilde{I}_{1}, \tilde{I}_{2}, \cdots, \tilde{I}_{m}\right], L=\left[\tilde{l}_{1}, \tilde{l}_{2}, \cdots, \tilde{l}_{m}\right], S=\left[\tilde{s}_{1}, \tilde{s}_{2}, \cdots, \tilde{s}_{m}\right]$, and $N=$ $\left[\tilde{n}_{1}, \tilde{n}_{2}, \cdots, \tilde{n}_{m}\right] \in \mathbb{R}^{n \times m}$, where $m$ is the number of slices, $n$ is the number of 
pixels in each slice, $\tilde{I}_{k}, \tilde{l}_{k}, \tilde{s}_{k}$, and $\tilde{n}_{k}$ are strung out of $I_{k}, l_{k}, s_{k}$ and $n_{k}$ into column vectors, $k=1,2, \cdots, m$. Although each $\widetilde{I}_{k}$ can be significantly different from others because of the different noise $\tilde{n}_{k}, \tilde{l}_{k}$ is expected to be the same for all $k$. The rank of $L$ is thus low. In low rank matrix completion, we want to solve $L$ from given $X$. Instead of relying on the cancel out of the noise by averaging, our low rank approach estimates the noise and relies on the estimation accuracy.

The rest of paper is organized as follows. In Section 2, we introduce the method including the preliminary speckle reduction using SRAD, the image registration by global and local alignment based on iterative diamond search, and the image recovery by low rank matrix completion. Section 3 shows the experimental results and is followed by conclusions in the last section.

\section{Method}

\subsection{Denoising}

Because of the inevitable eye movement, the raw OCT slices are usually not well aligned and registration is required. However, they are affected by large speckle noise which may dominate the image registration. In this paper, we propose to use SRAD [4 to have a preliminary noise reduction. Our tests show that SRAD is able to remove the noise though some details are removed. The basic principle of SRAD is as follows. Given an intensity image $I_{0}(x, y)$, the output image $I(x, y, t)$ is evolved according to following partial differential equations in SRAD:

$$
\left\{\begin{array}{l}
\partial I / \partial t=\operatorname{div}[c \cdot \nabla I] \\
I(t=0)=I_{0}
\end{array}\right.
$$

where $c$ denotes the diffusion coefficient computed from $I$.

\subsection{Image Registration}

In this paper, we propose to register the raw OCT slices based on their de-noised version obtained using the above SRAD algorithm. The image registration or alignment between two slices is done by a global alignment followed by a local alignment. In the global alignment step, a translation $(\Delta x, \Delta y)$ including both horizontal and vertical directions is applied on the entire slice. Taking $I_{i}$ as a reference, for each $I_{j}, j=1,2, \cdots, m$ and $j \neq i$, we find the alignment $\left(\Delta x_{j}, \Delta y_{j}\right)$ between $I_{i}$ and $I_{j}$ such that their difference is minimized.

In the local alignment step, an $A$-scan line (i.e., a column) or a group of neighboring $A$-scan lines from one $B$-scan slice are translated vertically (up or down) for best matching to the corresponding $A$-scan lines in a reference $B$-scan slice. As illustrated in Fig. 2, we divide a $B$-scan slice $B_{j}$ to non-overlapping $A$-scan group $A_{j, k}, k=1,2, \cdots, P$, where $P$ is the number of $A$-scan groups in $B_{j}$. Each group has $l A$-scan lines. For $A_{i, k}$ and $A_{j, k}$ from $B_{i}$ and $B_{j}$, find the vertical translation $\Delta x_{j, k}$ between them such that their error is minimized. In this paper, we use $l=8$. Since the eye movement is expected to be smooth, a 


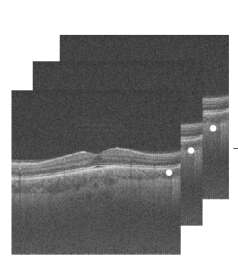

Input Slices

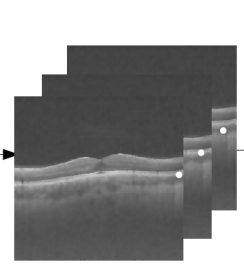

Denoised Slices

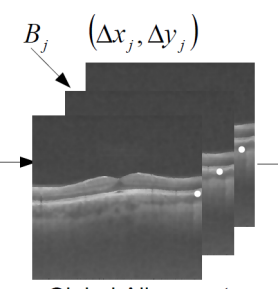

Global Alignment

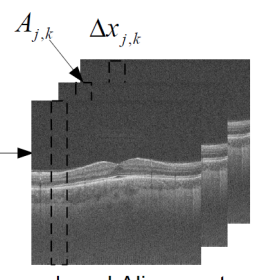

Local Alignment

Fig. 2. Illustration of Image Registration

smoothing process is applied to the vertical translations for lines in the same slice. The local alignment is done based on $A$-scans instead of other patches because each column is an outcome of an $A$-scan and the eye movement during the capture of one $A$-scan is minimal and ignored. By applying vertical alignment on $A$-scans, we protect the integrity of each $A$-scan.

In the above, a full search of the matching can be time consuming. In this paper, we use the iterative diamond search strategy [7] because of its efficiency and easy implementation. In the searching procedure, the algorithm iteratively searches among the neighboring points for the alignment between current and reference slice until the error in between is no longer decreasing. In the global alignment, the searching is done in both horizontal and vertical neighbors. In the local alignment, the searching is done in vertical neighbors only.

\subsection{Image Recovery}

After the image registration, a set of registered slices are obtained. Then they are vectorized and stacked to form matrix $X$, where $X=L+S+N$ as illustrated in Fig. 3. Low rank matrix completion is applied to compute the underlying clean image.

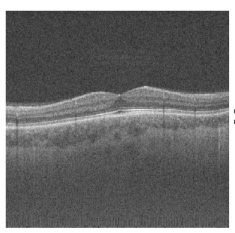

$X$

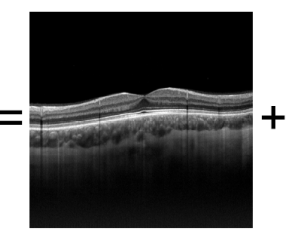

$L$

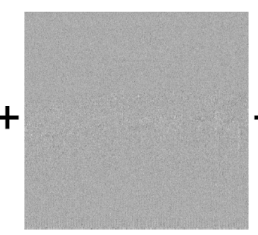

$S$

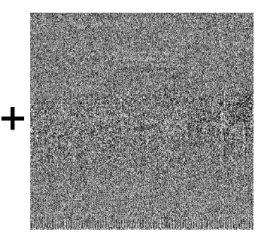

$N$

Fig. 3. Illustration of the model: each image shown here corresponds to a column in the matrix $X, L, S, N$, reshaped to a $2 \mathrm{D}$ image

Ideally, the clean images for all the slices are identical. In low rank approximation, $X$ is modelled as:

$$
X=L+S+N, \text { s.t., } \operatorname{rank}(L) \leq r, \operatorname{card}(S) \leq k
$$


The above decomposition is solved by minimizing the decomposition error:

$$
\begin{aligned}
\min _{L, S} & \|X-L-S\|_{F}^{2} \\
\text { s.t., } & \operatorname{rank}(L) \leq r, \operatorname{card}(S) \leq k
\end{aligned}
$$

Since there are two unknowns $L$ and $S$, the optimization problem above is solved by alternatively solving the following two sub-problems until convergence.

$$
\begin{aligned}
& L_{t}=\arg \min _{\operatorname{rank}(L) \leq r}\left\|X-L_{t-1}-S_{t-1}\right\|_{F}^{2} \\
& S_{t}=\arg \min _{\operatorname{card}(S) \leq k}\left\|X-L_{t-1}-S_{t-1}\right\|_{F}^{2}
\end{aligned}
$$

The problem in (4) can be solved using a singular value decomposition of $(X-$ $S_{t-1}$ ) as in robust principle component analysis (RPCA) 8]. However, RPCA is very slow. In this paper, we solve it using the power scheme modified bilateral random projection (BRP) 9, 10. We calculate the BRP of a matrix $\tilde{X}=\left(\left(X-S_{t-1}\right)\left(X-S_{t-1}\right)^{T}\right)^{q}\left(X-S_{t-1}\right)$. The BRP of $\tilde{X}$ is:

$$
Y_{1}=\tilde{X} A_{1}, Y_{2}=\tilde{X}^{T} A_{2},
$$

where $A_{1} \in \mathbb{R}^{m \times r}$ and $A_{2} \in \mathbb{R}^{n \times r}$. The approximation of $\left(X-S_{t-1}\right)$ with rank $r$ is then computed by

$$
L=Q_{1}\left[R_{1}\left(A_{2}^{T} Y_{1}\right)^{-1} R_{2}^{T}\right]^{\frac{1}{2 q+1}} Q_{2}^{T},
$$

where $Q_{1}, R_{1}, Q_{2}, R_{2}$ are obtained by $Q R$ decompositon of $Y_{1}$ and $Y_{2}$.

The problem in (5) is solved by updating $S_{t}$ via entry-wise hard thresholding of $X-L_{t}$ :

$$
S_{t}=\wp_{\Omega}\left(X-L_{t}\right),
$$

where $\wp_{\Omega}(Z)$ is a mapping to keep the largest $k$ entries of $|Z|$ and assign the rest as 0 . Detailed computation steps are summarized in the algorithm below.

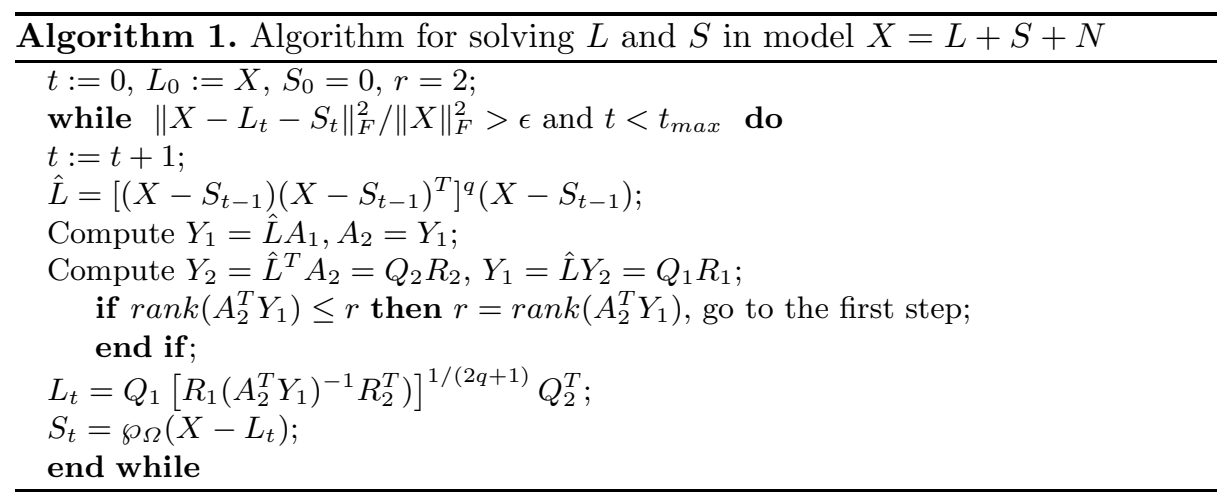




\section{$3 \quad$ Experimental Results}

In this paper, we conducted tests using 20 different subject eyes. For each subject eye, a line mode scan with 96 repeated slices are obtained. Each slice is an image with $992 \times 1024=1015808$ pixels. The matrix $X$ is a $1015808 \times 96$ dimensional matrix.

It is important to have an objective measurement to evaluate the performance of speckle noise reduction. As we do not have the ground truth image or original image for comparison, traditional measurement such as peak signal to noise ratio cannot be computed here. In this paper, we compute the contrast to noise ratio (CNR) that is commonly used in similar tasks [5], 11]. It measures the contrast between a set of regions of interest and a background region:

$$
C N R=\frac{1}{R} \sum_{r=1}^{R} \frac{\mu_{r}-u_{b}}{\sqrt{\left(\sigma_{r}^{2}+\sigma_{b}^{2}\right)}}
$$

where $\mu_{b}$ and $\sigma_{b}$ are the mean and variance of the same background region, and $\mu_{r}$ and $\sigma_{r}$ are the mean and variance of all the regions of interest. In this paper, we randomly obtain $20(R=20)$ regions of interest with $5 \times 5$ pixels from the retina layers in each image and one $5 \times 5$ background region.

To evaluate the performance of the proposed method, two methods denoted as 'Baseline' and 'Motion+Average' are compared with the proposed method. The 'baseline' denotes the state-of-the-art method which uses the sub-pixel registration and averaging approach as that in the current DRI OCT-1. In 'Motion+Average', we replace the sub-pixel registration in the 'Baseline' with the proposed registration while maintaining the averaging step. In the proposed method, we further replace the averaging with the BRP-based low rank matrix completion. Table 1 shows the average CNR computed from 20 images with the number of re-scanned slices $m=96$. With a preliminary speckle reduction, the proposed diamond search based registration helps improve the CNR. The proposed method further improves the CNR by replacing averaging with low rank matrix completion. This is because the low rank reconstruction model is less sensitive to large noise from one or several slices compared with averaging as the latter cannot avoid it while the former is able to handle it better by estimation.

Table 1. Performance (CNR) by various methods

\begin{tabular}{c|c|c|c}
\hline Method & Baseline & Motion + Average & Proposed \\
\hline CNR & 13.78 & 14.07 & 15.65 \\
\hline
\end{tabular}

To evaluate the robustness of the proposed methods for different number of slices, we further conducted tests for the number of slices $m$ from 16 to 96 with a step of 16. Fig. 4 plots the average CNRs for the three methods. The results show that the proposed method outperforms the baseline method consistently 


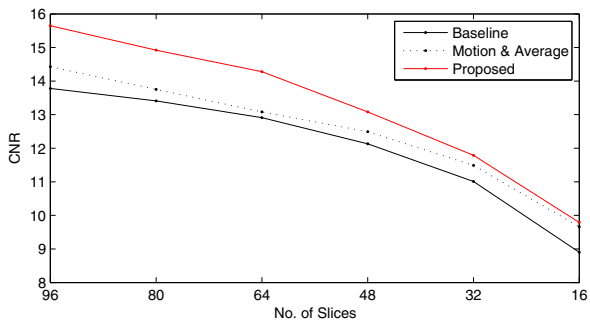

Fig. 4. Performance comparison for different number of reference images
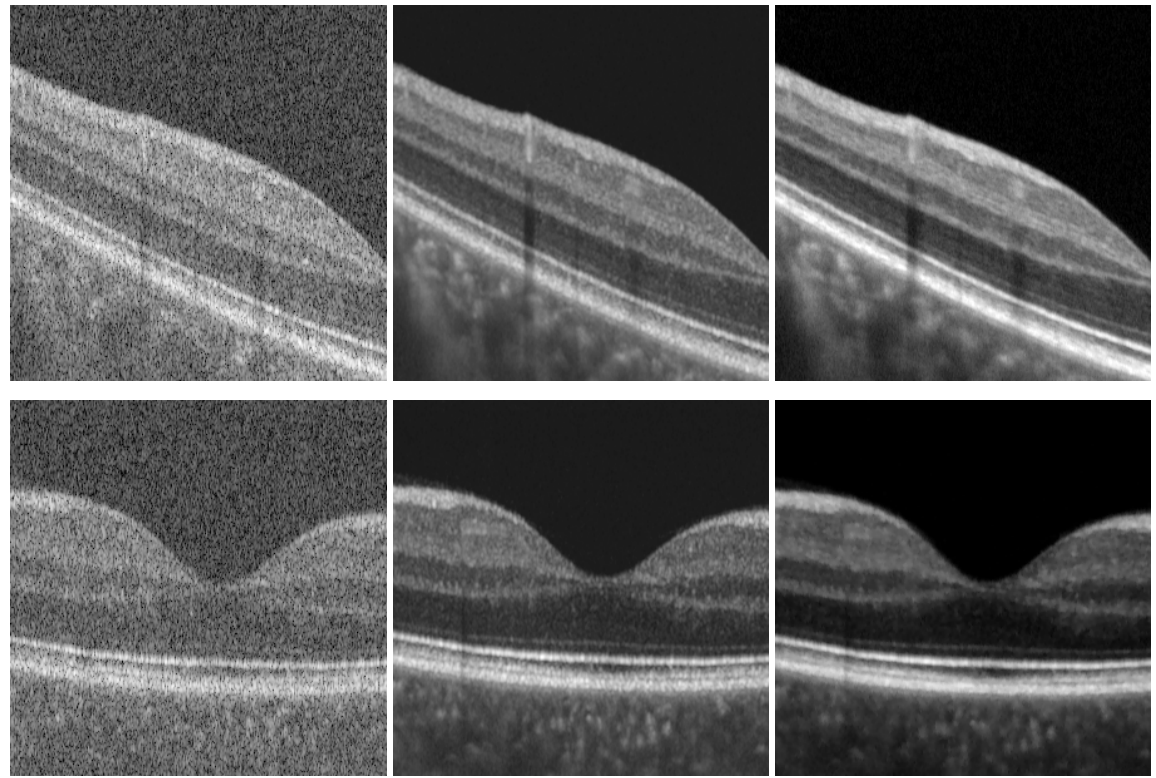

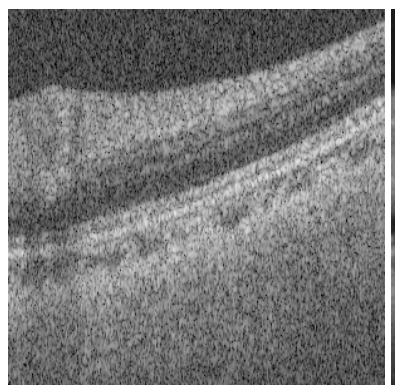

(a) Raw slice

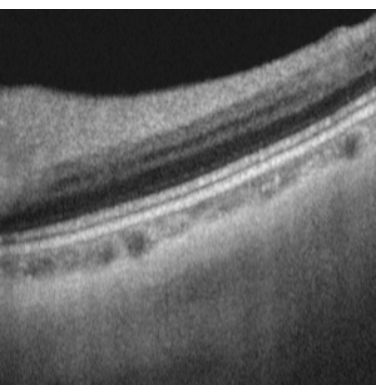

(b) Baseline

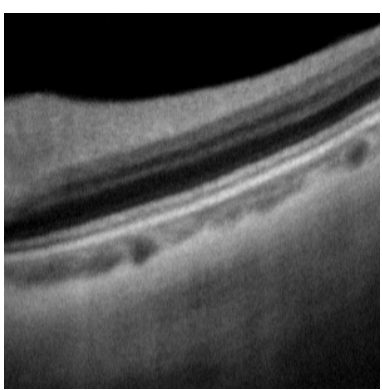

(c) Proposed

Fig. 5. Sample results: (a) $300 \times 300$ regions cropped from raw slices for highlight, (b) and (c) are the corresponding portion of images obtained by the baseline and proposed method 
but the improvement decreases with $m$. In addition, Fig. 5 shows three sample results to visualize the difference between the baseline and proposed method. As we can see, the images obtained from the proposed method show less speckle noise and better structure details than those from the baseline method.

In this paper, we solve the low rank matrix completion using power scheme modified BRP. It is faster than traditional algorithms such as RPCA with similar recovered results. It takes about $5 \mathrm{~s}$ for one iteration of $L_{t}$ to recover a image of $992 \times 1024$ pixels from 96 slices in a dual core $3.0 \mathrm{GHz}$ PC with $3.25 \mathrm{~GB}$ RAM in MATLAB. For the same task, RPCA [8] requires 100s.

\section{Conclusions}

In this paper, we propose a novel algorithm for specke noise reduction in swept source OCT images. Instead of using a sub-pixel registration algorithm that minimizes the mean square intensity difference, we propose to register the raw OCT slices using fast diamond search from speckle reduced slices. In addition, averaging is replaced by low rank matrix completion using power scheme modified BRP. The main difference is that the former averaging approach relies on the cancelling out of speckle noise while the later low rank algorithm estimates the noise and relies on its accuracy in the estimation. Our experimental results show that the proposed method outperforms the currently used baseline method. The limitation of current method is that it requires a number of repeated scans from the same location.

\section{References}

1. Lopes, A., Touzi, R., Nesby, E.: Adaptive speckle filter and scene heterogeneity. IEEE Trans. Geosci. Remote Sens. 28, 992-1000 (1990)

2. Lim, S.J.: Two-Dimensional Signal and Image Processing. Prentice Hall (1990)

3. Perona, P., Malik, J.: Scale space and edge detection using anisotropic diffusion. IEEE Trans. Pattern Anal. Machine Intell. 12 (1990)

4. Yu, Y., Acton, S.T.: Speckle reducing anisotropic diffusion. IEEE Trans. Image Processing 11, 1260-1270 (2002)

5. Szkulmowski, M., Wojtkowski, M.: Averaging techniques for oct imaging. Opt. Express 21, 9757-9773 (2013)

6. Thévenaz, P., Ruttimann, U.E., Unser, M.: A pyramid approach to subpixel registration based on intensity. IEEE Trans. Image Processing 7, 27-41 (1998)

7. Zhu, S., Ma, K.: A new diamond search algorithm for fast block-matching motion estimation. IEEE Trans. Image Processing 9, 287-290 (2000)

8. Candès, E., Recht, B.: Exact matrix completion via convex optimization. Commun. ACM 55, 111-119 (2012)

9. Zhou, T., Tao, D.: Bilateral random projections. In: IEEE Int. Symp. on Information Theory, pp. 1286-1290 (2012)

10. Zhou, T., Tao, D.: Godec: Randomized low-rank \& sparse matrix decomposition in noisy case. In: Proc. of the 28th Int. Conf. on Machine Learning, pp. 33-40 (2011)

11. Rogowaka, J., Brezinski, M.E.: Evaluation of the adaptive speckle suppression filter for coronary optical coherence tomography imaging. IEEE Trans. Medical Imaging 19, 1261-1266 (2000) 